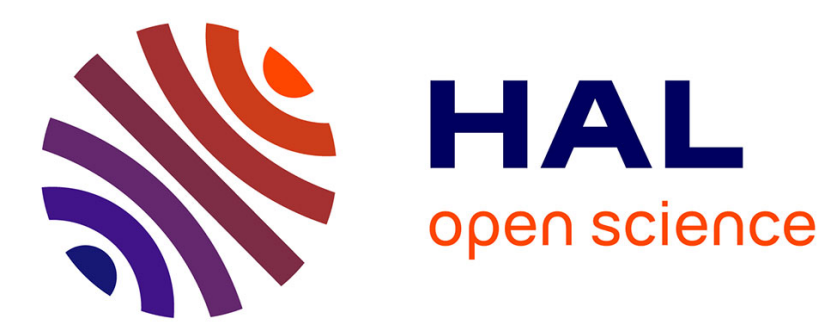

\title{
Réflexions sur les biens communs
}

Carol Rose, Judith Rochfeld, Emma Guernaoui, Jean-Baptiste Roche, Thomas Perroud

\section{To cite this version:}

Carol Rose, Judith Rochfeld, Emma Guernaoui, Jean-Baptiste Roche, Thomas Perroud. Réflexions sur les biens communs. Droit \& societe: theorie et sciences sociales du droit., 2021, pp.753-769. hal-03137621

\section{HAL Id: hal-03137621 \\ https://hal.science/hal-03137621}

Submitted on 28 Apr 2021

HAL is a multi-disciplinary open access archive for the deposit and dissemination of scientific research documents, whether they are published or not. The documents may come from teaching and research institutions in France or abroad, or from public or private research centers.
L'archive ouverte pluridisciplinaire HAL, est destinée au dépôt et à la diffusion de documents scientifiques de niveau recherche, publiés ou non, émanant des établissements d'enseignement et de recherche français ou étrangers, des laboratoires publics ou privés. 


\title{
Réflexions sur les biens communs
}

\author{
Carol M. Rose
}

Yale Law School, 127 Wall Street, New Haven, CT 06511, États-Unis.

<carol.rose@yale.edu>

* Texte traduit de l'anglais par Emma Guernoui et Jean-Baptiste Roche

Résumé

Un regard rétrospectif sur les recherches concernant les biens communs initiées par Elinor Ostrom, et largement suscitées en réaction contre le fameux article de Garrett Hardin sur la "Tragédie des biens communs", permet de mesurer le rôle majeur qu'ont joué ces deux figures dans le développement du thème des communs. Les observations critiques n'ont certes pas manqué mais elles ont contribué à dégager de nouvelles questions et de nouvelles perspectives, enrichissant ainsi le catalogue des pistes de recherche et remettant en cause des vérités parfois prises pour argent comptant.

Accès ouvert-Environnement-Propriété-Ressources - Science citoyenne.

\section{Summary Thinking About the Commons}

A retrospective look at the research on commons initiated by Elinor Ostrom, and largely prompted in reaction to Garrett Hardin's famous article on the "Tragedy of the commons", allows us to measure the major role played by these two figures in the development of the theme of commons. As expected criticism has been made, though it contributed to the identification of new questions and new perspectives, thus enriching the catalogue of research approaches and challenging truths that are sometimes taken at face value.

Citizen science - Environment-Open access - Property-Resources. 


\section{Présentation du texte de Carol Rose}

Dans le cadre d'un projet de recherches financé par la Mission de recherche "Droit et Justice " et intitulé "L'échelle de communalité - Propositions de réformes pour intégrer les biens communs en droit", dirigé par Judith Rochfeld, Carol Rose a bien voulu accepter de débattre des propositions émises et de prononcer une leçon inaugurale au printemps 2019, traduite ici, grâce au travail d'Emma Guernaoui et de Jean-Baptiste Roche.

Aujourd'hui professeure émérite à l'Université de Yale, Carole Rose est l'autrice de nombreux travaux qui constituent une référence essentielle pour la réflexion critique sur le droit de propriété et la réhabilitation du concept de commun. Son article «La comédie des communs ${ }^{1}$ fut publié en 1986. Il marqua un jalon important vers le retour à une vision plus équilibrée des communs avant la publication en 1990 de l'ouvrage majeur d'Ostrom, Governing the Commons.

Ces «communs" proposés par Carole Rose étaient toutefois d'un type particulier se rapprochant davantage, pour un public français, d'un domaine public. Dans cette leçon, elle dresse un bilan et formule des pistes de réflexions sur l'avenir de la recherche sur les communs - telle, par exemple, la question de l'accès ouvert (" open access »), trop peu étudiée selon elle. Ce ne sont là que quelques-uns des problèmes fascinants qui demeurent à explorer pour tenter de refonder le droit de propriété dans nos sociétés.

Judith Rochfeld et Thomas Perroud

Quel est l'état de la réflexion académique sur les biens communs? J'ai l'intention d'explorer ici au moins quelques unes des tendances actuelles de la recherche sur ce sujet, principalement, mais pas exclusivement, parmi les chercheurs américains. Afin d'aborder ces questions, j'organiserai mes remarques relatives à la réévaluation et au suivi de la recherche autour de deux des grands noms de l'étude des biens communs. Il s'agit de Garrett Hardin, dont l'article sur la «Tragédie des biens communs » (1968) 2 a cristallisé l'intérêt moderne pour les caractéristiques des ressources communes; et d'Elinor Ostrom, dont le Governing the Commons (1990) ${ }^{3}$ a donné une réponse à $\mathrm{G}$. Hardin, à savoir que les biens communs ne doivent en rien être tragiques, notamment dans le cas où une communauté peut gérer un patrimoine de portée limitée. Les travaux de E. Ostrom sur les biens communs ont suscité un afflux de recherches sur les questions de propriété commune à la fois passées et contemporaines, et ses propres analyses sur ces sujets étaient beaucoup plus étendues et systématiques que les remarques relativement désinvoltes de G. Hardin

1. Carol Rose, "The Comedy of the Commons: Custom, Commerce, and Inherently Public Property", University of Chicago Law Review, 53 (3), 1986, p. 711-781.

2. Garrett Hardin, « The Tragedy of the Commons », Science, 3859, 1968, p. 1243-1248.

3. Elinor Ostrom, Governing the Commons: The Evolution of Institutions for Collective Action, Cambridge : Cambridge University Press, 1990. 
qu'elle a elle-même vivement critiqué. En effet, une grande partie du réexamen de la «Tragédie » de G. Hardin a été plutôt critique, s'inspirant peut-être d'E. Ostrom. Mais le travail de cette dernière a également fait l'objet d'un réexamen portant tant sur le plan de la méthode que sur le fond.

Dans cet essai, je reviendrai d'abord sur le réexamen historique récent de la «Tragédie» de G.Hardin, dont l'une des principales critiques est que ses idées n'étaient en réalité pas si originales que cela. J'aborderai ensuite quelques relectures récentes des travaux de E. Ostrom sur la gestion limitée des biens communs, travaux qui restent largement célébrés mais aussi critiqués au moins légèrement pour des raisons de flou méthodologique et de contenu économique et politique. Je parlerai ensuite de ce qui me semble être le principal défi pour les membres de l'école de E. Ostrom sur les biens communs, à savoir les situations dans lesquelles ces biens communs ne se réfèrent pas à des biens communs limités relativement fermés mais plutôt en libre accès. Par exemple, les communications par Internet et les problèmes environnementaux à grande échelle, où les questions de gouvernance se posent de façon pressante. Je terminerai en mentionnant certains des nombreux sujets que j'ai laissés en suspens et qui sont pourtant des orientations pour l'avenir de la recherche dans ce domaine important.

\section{Histoire et tragédie des biens communs de Garrett Hardin}

Le terme commons est assez ancien dans la langue anglaise, mais jusqu'au milieu du siècle dernier, il avait un sens assez restreint. Tout d'abord, le commun se référait aux champs agricoles communs qui faisaient partie de l'économie rurale médiévale; normalement, ces champs étaient ouverts aux habitants des villages et utilisés pour le pâturage du bétail, la coupe du bois et d'autres activités extractives de bas niveau. Deuxièmement, les biens communs désignaient les lieux de rencontre dans les villes ou les villages, où les habitants pouvaient venir pour discuter de questions politiques, organiser des festivals locaux ou participer à d'autres activités à l'échelle du village ou de la ville. Troisièmement, et par extension, les communs faisaient référence à des espaces communs dans des bâtiments comme les structures universitaires, où les étudiants pouvaient manger, se détendre et rencontrer des amis.

L'utilisation plus généralisée de l'expression « les biens communs » comme raccourci pour désigner toutes sortes d'activités regroupant plusieurs personnes, souvent avec des problèmes d'organisation contrariants, découle du très célèbre article de Garrett Hardin paru en 1968 dans la revue Science, intitulé «La tragédie des biens communs ".

Dans ce texte, qu'il a écrit principalement pour mettre en garde contre les dangers de la surpopulation, G. Hardin a appliqué le terme "bien commun " à une grande variété de scénarios dans lesquels certaines ressources sont ouvertes à tous, avec peu de contraintes quant à leur utilisation. Selon lui, dans ce scénario, la tendance de chaque individu est d'utiliser la ressource pour maximiser son propre intérêt immédiat tout en négligeant l'investissement ou l'effort qui pourrait conserver la ressource pour les autres ou pour une utilisation commune future. Selon la 
thèse de G. Hardin, chaque individu calcule rationnellement qu'il profite de tous les gains de sa propre utilisation, mais partage les pertes avec tous les autres utilisateurs. En suivant cette logique, tous les utilisateurs épuisent ensemble la ressource commune. Pour lui, le seul moyen d'empêcher cette tragédie est la coercition gouvernementale (qu'il appela plus tard Léviathan), ou la division des biens communs en propriété privée ${ }^{4}$.

La préoccupation immédiate de G. Hardin, bien sûr, était ce qu'il considérait comme une crise de surpopulation. Il a exprimé le rapport au bien commun par l'analogie entre les utilisateurs d'un bien commun non réglementé quel qu'il soit et les éleveurs de bétail. Mais il a donné d'autres exemples, variés, des types de biens communs. Certes, il a surtout parlé des bergers dont les nombreux troupeaux pâturaient à outrance un champ commun, mais il a aussi cité la surpêche et la chasse excessive, les automobiles à la recherche de places de stationnement, la pollution atmosphérique et la pollution de l'eau, parmi d'autres exemples.

L'article de G. Hardin employait donc le terme « biens communs » pour désigner de façon très générale les problèmes d'action collective et, depuis sa parution, un grand nombre d'autres auteurs ont abordé la problématique des " communs » dans ce sens plus général, certains adoptant la théorie de la surutilisation et du sousinvestissement, d'autres la contestant. Le cinquantième anniversaire de la parution de l'article de G. Hardin, en 2018, a remis l'article lui-même sous les feux de la rampe, ainsi que la conception générale des biens communs de son auteur. L'historien du droit David Schorr, de la Faculté de droit Buchman de l'Université de Tel Aviv et du Cegla Center for Interdisciplinary Research in Law, a profité de l'occasion pour organiser une conférence rétrospective sur cet article, suivie d'un symposium publié dans Theoretical Inquiries in Law du Centre Cegla 5 .

Le colloque Cegla est un bon point de départ pour aborder les réflexions récentes sur les biens communs, et en particulier sur l'histoire du concept. Plusieurs contributions au colloque soulignent que G. Hardin ne disait pas grand-chose de nouveau dans ses observations sur la fragilité des ressources mises en commun. En effet, Stuart Banner de l'UCLA Law School décrit l'idée de G. Hardin comme étant banale, et ilobserve que les penseurs depuis Aristote ont discuté du problème de la surutilisation et du sous-investissement dans les ressources communes. Si S. Banner suit l'idée de la surutilisation à travers divers écrits médiévaux et modernes, il souligne particulièrement la contribution de l'économiste du XIX siècle William Forster Lloyd. W.Lloyd lui-même répondait à l'argument de Thomas Malthus selon lequel la surpopulation découlerait du manque général de maîtrise de soi, et son commentaire non seulement anticipait les arguments de G.Hardin sur la surpopulation, mais utilisait précisément l'exemple du bétail paissant en plein champ que G. Hardin avait si bien mobilisé dans la «Tragédie». En effet, G. Hardin lui-même a reconnu

4. Ibid.; ID., « Political Requirements for Preserving Our Common Heritage », in Howard P. BroKAW, Wildlife in America, Washington D.C. : Council of Environmental Quality, 1978, p. 310-317, p. 314.

5. V. le volume intitulé « The Tragedy of the Commons at 50: Context, Precedents, and Afterlife ", Theoretical Inquiries in Law, 19 (2), 2018, p. 395-745. Le professeur D. Schorr m'a proposé d'être corédactrice, mais il a fait de loin la majeure partie du travail de supervision de l'évènement, d'organisation et d'édition de l'évènement. 
qu'il avait emprunté cet exemple à Lloyd, bien qu'il soit resté très avare quand il s'est agi de reconnaître ce qu'il devait à son prédécesseur. Selon S. Banner, la réussite principale de G. Hardin fut non pas de fournir une analyse des ressources communes, lesquelles avaient été observées pendant des siècles, mais plutôt d'avoir donné un nom accrocheur pour leur caractère problématique, un nom qui nous a accompagné depuis l'article de G. Hardin ${ }^{6}$.

Comme S. Banner et d'autres l'ont souligné, G. Hardin a négligé ou ignoré un certain nombre d'autres efforts, antérieurs et contemporains, sérieux pour reconnaître et régler le problème de la surutilisation. L'article de l'économiste des ressources Scott Gordon 7, qui, en 1954, avait analysé le problème des biens communs dans le contexte des pêcheries et conclu que la surutilisation résultait d'une divergence entre le coût moyen et le coût marginal de l'utilisation des ressources communes, était peut-être l'un des plus remarquables. Cette divergence s'explique par le fait que chaque pêcheur supplémentaire a calculé ses coûts sur la base de la moyenne de tous les pêcheurs précédents, sans tenir compte de la pression croissante sur les ressources que chaque nouveau pêcheur ajouterait. Et S. Gordon n'était pas le seul théoricien à l'époque. En 1965, l'économiste Mancur Olsen a utilisé la théorie de l'action collective pour analyser l'activité politique et en particulier la dépense excessive systématique des fonds publics dans un gouvernement représentatif. En 1967, un an à peine avant la publication de la "Tragédie " de G. Hardin, l'économiste Harold Demsetz avait utilisé la surutilisation des ressources communes pour illustrer le phénomène d'externalité, dans lequel les utilisateurs agissent sans tenir compte des effets sur les autres (ou sur eux-mêmes à long terme, dans le cas des effets externes communs). G. Hardin ne semblait pas être au courant du travail de ces théoriciens.

Certes, ces travaux ont été écrits par des économistes plutôt que par des biologistes comme l'était G. Hardin. Mais on aurait pu s'attendre à ce qu'il accorde plus d'attention aux documents sur les pêcheries que l'historien Harry Scheiber ${ }^{8}$ cite dans le symposium Cegla. Comme H. Scheiber le décrit longuement, dans les décennies qui ont précédé l'apparition de la tragédie, le problème de l'épuisement des stocks de poissons avait donné lieu à de nombreuses conférences, articles (dont celui de S. Gordon) et mesures pratiques pour lutter contre la surpêche, mais G. Hardin n'en a nullement tenu compte ${ }^{9}$.

L'historien du droit David Schorr est à peine moins critique à l'égard de la « Tragédie» de G. Hardin, la situant dans une sorte de tradition méta-historique qu'il décrit comme fonctionnant par stades («stadial»). Dans ce type d'analyse plutôt démodé, les historiens décrivent les sociétés comme évoluant par stades, de la société

\footnotetext{
6. Stuart BANNER, «The Banality of the Commons: Efficiency Arguments Against Common Ownership Before Hardin », ibid., p. 395-409.

7. H. Scott GORDON, "The Economic Theory of a Common-Property Resource: The Fishery ", Journal of Political Economy, 62 (2), 1954, p. 124-142.
}

8. Harry N. SCHEIBER, «The Commons Discourse on Marine Fisheries Resources: Another Antecedent to Hardin's ", Theoretical Inquiries in Law, 19 (2), 2018, p. 489-506.

9. Ibid. 
primitive à l'agriculture, puis au commerce, à la fabrication et finalement à la technologie moderne. D. Schorr soutient que les analyses des ressources couramment mises en commun, y compris celles de G. Hardin, suivent un tel modèle dans lequel les auteurs décrivent la progression d'un stade à un autre. Dans le cas de G. Hardin, les stades sont quelque peu laconiques: les biens communs non réglementés au stade primitif, et à celui plus avancé, soit la propriété privée, soit le commandement du gouvernement. Selon D. Schorr, le schéma le plus général consiste à décrire plusieurs stades, généralement au nombre de quatre: d'abord le bien commun n'est pas gouverné, puis il fait l'objet d'une exclusion tribale, laquelle est suivie de mesures réglementaires, et enfin il est divisé en propriété privée 10.

Je connais bien cette progression passée de mode, car D. Schorr utilise à titre d'exemple un article que j'ai écrit en 1991 sur les différents stades de la gestion environnementale. Pour ceux qui pourraient être intéressés, je présente ma position en note de bas de page 11 .

L'élément principal est que les chercheurs qui ont récemment réexaminé la «Tragédie des communs » de G. Hardin d'un point de vue historique ont généralement été assez critiques, et leurs critiques ont été adoucies principalement, sinon entièrement, par le fait que sa principale préoccupation était de se livrer à des récriminations contre la surpopulation. Compte tenu de cette préoccupation centrale, on ne s'attendait peut-être pas à ce que G. Hardin en sache beaucoup sur les travaux d'autres disciplines et sur ce qui était pour lui une analogie relativement improvisée. Mais un autre type de critique est que G. Hardin avait tout simplement tort quelles qu'aient pu être ses thèses les plus importantes. Cette critique nous amène à Elinor Ostrom et son école.

10. David B. SCHORR, «Savagery, Civilization, and Property: Theories of Societal Evolution and Commons Theory", ibid., p. 507-531.

11. Mon article était «Rethinking Environmental Controls: Management Strategies for Common Resources ", Duke Law Journal, 1, 1991, p. 1-38 dans lequel j'ai soutenu qu'au fur et à mesure que les pertes en commun se produisent, les gens sont susceptibles de changer de stratégie, passant de l'inaction à la limitation de l'accès à un groupe, à la réglementation du comportement et à la création de droits de propriété individuels plus précis (comme pour les permis de pollution). C'est certainement par stades (stadial), mais l'article de D. Schorr me donne l'occasion d'en dire plus sur cette analyse. Tout d'abord, au niveau le plus général, il n'est pas possible, en langage humain, de discuter des différentes tendances sans les diviser en catégories, qu'on les appelle ou non stades. Deuxièmement, si certaines étapes peuvent être entièrement artificielles (au XVII ${ }^{\mathrm{e}}$ siècle, XVIII ${ }^{\mathrm{e}}$ siècle), d'autres, comme les stratégies de gestion de l'environnement, peuvent présenter de réelles différences de techniques et de conséquences, de sorte qu'un passage de l'une à l'autre est visible pour les personnes concernées. Par exemple, on peut citer l'adoption de permis d'émissions échangeables comme stratégie de réduction de la pollution en vertu des modifications apportées en 1990 à la loi sur la qualité de l'air, qui a suscité de vives discussions en raison de l'abandon d'un modèle antérieur de réglementation comportementale (le soi-disant commander et contrôler). Troisièmement, enfin, si les discussions sur les réponses environnementales peuvent suggérer des étapes, elles ne sont pas nécessairement unidirectionnelles, mais répondent plutôt à des conditions de rareté ou de demande, allant dans un sens avec une plus grande rareté/demande mais dans le sens contraire si les conditions sont remplies. Outre mon article de 1991, un autre exemple est celui de Terry ANDERson et de P. J. HiLL, dans lequel ils décrivent bien l'évolution des droits de propriété dans l'Ouest américain ( The Evolution of Property Rights: A Study of the American West », Journal of Law and Economics, 18 (1), 1975, p. 163-180) et qui ont observé un assouplissement de la réglementation à mesure que la demande diminuait pour certaines ressources, par exemple les chevaux. 


\section{Sciences sociales : Elinor Ostrom et les biens communs à gestion limitée}

Elinor Ostrom a littéralement surgi parmi les chercheurs travaillant sur les biens communs avec son livre de 1990, Governing the Commons, un ouvrage qui, depuis, reste essentiel aux recherches sur les communs 12 . Et elle était plus que prête à combattre l'idée que les communs sont tragiques, ou plus particulièrement, que les seuls remèdes aux problèmes des communs sont la coercition gouvernementale d'une part, ou la propriété privée d'autre part. Dans Governing the Commons, comme dans presque tout ce qu'elle a écrit par la suite ${ }^{13}$, elle commençait par inclure un ou deux paragraphes reprochant à Garrett Hardin d'affirmer que les ressources communes ne pouvaient être gérées que par ces deux voies. Au contraire, a-t-elle fait valoir, l'expérience a montré que les gens trouvaient souvent des moyens d'organiser et de gérer les ressources communes grâce à des méthodes de gouvernance communautaires plutôt que par le Léviathan étatique ou par la propriété privée.

Comme l'article de G. Hardin, les travaux de E. Ostrom prolongeaient ceux d'un certain nombre d'autres chercheurs, mais dans son cas, il s'agissait de chercheurs qui avaient critiqué la thèse de G. Hardin pendant la décennie ou les deux décennies précédant son livre révolutionnaire, ou qui avaient eux-mêmes étudié des programmes de gestion communautaire. En 1985, par exemple, Susan Buck Cox avait publié un ouvrage provocateur intitulé No Tragedy of the Commons ${ }^{14}$ citant un certain nombre de programmes de gestion des biens communs ayant connu du succès au cours de l'histoire. En 1987, James Acheson et Bonnie McCay avaient également fait paraître une compilation d'études des ressources communautaires intitulée The Question of the Commons ${ }^{15}$. Contrairement à G. Hardin, E. Ostrom a non seulement reconnu les travaux de ses prédécesseurs, mais elle les a aussi défendus. Dans son œuvre intitulée Governing the Commons, elle a dressé sa propre liste de "principes d'aménagement» qui encourageaient une gestion communautaire réussie des biens communs, mais elle s'est inspirée à maintes reprises d'exemples tirés, entre autres, des études de Meg McKeon sur les pratiques forestières communes des villages japonais, de la description des communautés alpines et de leurs règles communes de pâturage, et des travaux de A. Maass et R. L. Anderson sur les systèmes communautaires d'irrigation en Espagne qui remontent à l'ère des musulmans.

Elinor Ostrom, avec son mari Vincent Ostrom, avaient déjà fondé le Workshop in Political Theory and Policy Analysis 16 à l'Université de l'Indiana, mais après l'apparition de Governing the Commons, ce Workshop est devenu un véritable incubateur pour les études sur les biens communs à travers le monde. Bon nombre de

12. Elinor Ostrom, Governing the Commons: The Evolution of Institutions for Collective Action, op. cit.

13. Voir, par exemple, Elinor Ostrom, Joanna Burger, Christopher B. Field, Richard B. NorgaARD et David POLICANSKY, « Revisiting the Commons: Local Lessons, Global Challenges », Science, 284, 5412, 1999, p. 278-282.

14. Susan B. Cox, « No Tragedy of the Commons », Environmental Ethics, 7 (3), 1985, p. 49-61.

15. James M. ACHESON et Bonnie J. MCCAY, The Question of the Commons: The Culture and Ecology of Communal Resources, Tucson : University. of Arizona Press, 1996.

16. Atelier de théorie politique et d'analyse des politiques [NdT]. 
ses étudiants et associés ont étudié le type de gestion communautaire traditionnelle des ressources qui domine les exemples dans Governing the Commons. Mais à partir de 2000, E. Ostrom a elle-même commencé à enrichir ses méthodes, d'abord ses «principes d'aménagement » et plus tard son " analyse et développement institutionnels » (IAD) pour des sujets plus contemporains. Elle s'est particulièrement intéressée à l'organisation sociale de la diffusion technologique moderne de l'information et a parfois joint ses forces à celles de ce que l'on pourrait appeler vaguement les «critiques copyleft de la propriété intellectuelle", généralement des juristes et des activistes qui ont renoncé à s'approprier des réalisations intellectuelles et ont souvent assimilé l'IP ("Intellectual Property») au processus d'enclosure des biens communs dans l'Angleterre moderne ${ }^{17}$.

Compte tenu de la générosité de E. Ostrom à citer et à promouvoir le travail des autres, il est tout à fait approprié et peut-être pas surprenant qu'en plus de nombreux prix dans sa spécialité, la science politique, son travail ait reçu de nombreux éloges de la part de chercheurs et ce dans de nombreux domaines. Le prix Nobel d'économie lui a été décerné en 2009 avec Oliver Williamson. Mais le travail de E. Ostrom n'a pas cessé de recevoir des éloges depuis sa mort en 2012. Par exemple, Lee Anne Fennell, Professeure de droit à l'Université de Chicago, a écrit un article très élogieux intitulé "Ostrom's Law" dans une rétrospective sur le travail de E. Ostrom en 2011. De plus, l'article de L. A. Fennell décrivant la loi d'Ostrom selon laquelle «tout ce qui fonctionne en pratique peut fonctionner en théorie » est devenu l'une des contributions les plus citées du journal en ligne dans lequel il est publié 18 .

Mais, au cours des dernières décennies, les chercheurs qui s'intéressent aux questions sur les biens communs ont également exprimé des hésitations et des préoccupations critiques au sujet de l'approche de E. Ostrom en matière de gestion des ressources. Une mise en garde importante a été lancée par le politologue Arun Agrawal, dont le travail sur la foresterie communale en Inde fait partie intégrante de la littérature courante de l'école Ostrom. En 2002, dans un volume édité par E. Ostrom elle-même et certains de ses associés, A. Agrawal a fait l'éloge de l'explosion de la recherche sur la propriété commune pour avoir dissipé l'idée que les biens communs étaient simplement une relique d'une époque révolue, et pour avoir montré que la gestion communautaire continue d'être un aspect vital des pratiques durables actuelles ${ }^{19}$. Ces résultats de recherche mettent en garde contre la tendance à la privatisation et à la gestion centrale de l'État dans l'économie moderne du développement.

Mais A. Agrawal a également observé que les nombreuses études de cas des pratiques locales n'accordaient généralement pas suffisamment d'attention au contexte social, institutionnel et technologique. Plus grave encore peut-être, ces études

17. James BoyLe, "The Second Enclosure Movement and the Construction of the Public Domain ", Law and Contemporary Problems, 66 (1-2), 2003, p. 33-74.

18. Lee Ann FenNell, "Ostrom's Law: Property Rights in the Commons», International Journal of the Commons, 5 (1), 2011, p. 9-27.

19. Arun Agrawal, "Common Resources and Institutional Sustainability ", in National ReSEARCH CounCIL. Committee on the Human Dimensions of Global Change (ed.), The Drama of the Commons, Washington: National Academy Press, 2002, p. 41-86. v. <https://www.nap. edu/read/10287/chapter/4>. 
ont cité de nombreux facteurs de réussite différents, et apparemment en interaction, pour expliquer le succès du partage des communs mais elles ne sont parvenues à aucune théorie cohérente pour expliquer leur gestion viable et durable 20 .

En fait, dans ces études sur les biens communs, le succès en soi semblait signifier la longévité, l'évitement de l'épuisement des ressources et ce qui a été décrit comme des résultats équitables ${ }^{21}$. Il serait tout à fait possible d'identifier le succès comme autre chose, par exemple, une plus grande productivité ou une version différente des résultats équitables, j'en dirai davantage plus loin dans mon exposé. On pourrait dire que l'idée générale des commentaires de A. Agrawal était que la loi d'Ostrom ("ce qui fonctionne dans la pratique peut aussi fonctionner en théorie ") n'avait pas encore été promulguée.

Il semblerait qu'elle ne le soit toujours pas, ouvrant la voie à une prise de contrôle du Workshop d'Ostrom à l'Université de l'Indiana par la science économique : le Président du conseil était jusqu'à récemment un économiste, Lee Alston, et ce même conseil comprend Dean Lueck, un autre économiste. Tous deux s'intéressent certes au développement du monde réel et aux questions environnementales, mais il est clair qu'ils abordent le sujet en économistes en privilégiant une approche microéconomique 22 .

\section{La critique des communs limités et le tournant de l'accès ouvert}

Tandis qu'au départ E. Ostrom et ses partisans se concentraient sur les modes d'organisation des communautés responsables des communs, un certain nombre de chercheurs remarquaient que ce type d'organisation pouvait rencontrer certains problèmes. L'ouvrage de Robert Ellickson, Order Without Law ${ }^{23}$, est paru un an après Governing The Commons de E. Ostrom et était consacré à l'étude des conceptions et coutumes des communautés fondées sur des normes pour gérer l'utilisation de ressources qui se chevauchent. R. Ellickson a utilisé comme exemple principal les pratiques d'une communauté d'éleveurs dans le nord de la Californie intervenant en dehors de la structure légale formelle et parfois même en contradiction avec celle-ci. Mais R. Ellickson a soulevé un point qui n'était pas tellement pris en compte dans la littérature relative aux communs de E. Ostrom: les normes communautaires n'étaient tout simplement pas en mesure de résoudre certains problèmes. Les éleveurs du comté ont trouvé des méthodes assez efficaces pour s'occuper des bovins s'égarant d'un ranch à un autre, mais dans les cas où les bovins erraient sur les routes et entraient en collision avec des automobiles, c'est le droit officiel qui était appliqué, en particulier lorsque les accidents étaient graves 24 .

20. Ibid., p. 42-45, 56-58, 64-67, 71 .

21. Ibid., p. 52.

22. Le conseil compte d'autres membres dont un spécialiste du «développement global » et deux chercheurs en propriété intellectuelle.

23. Robert C. ElLICKSON, Order without Law: How Neighbors Settle Disputes, Cambridge : Harvard University Press, 1991.

24. Ibid., p. 82-103. 
Au cours des années suivantes, d'autres chercheurs ont prolongé les observations de R. Ellickson concernant la faiblesse potentielle des normes communautaires à travers l'étude de problèmes à plus grande échelle. J'ai moi-même constaté à plusieurs reprises dans les années 1990 et 2000 que les communautés traditionnelles ne maîtrisent pas bien les problèmes de ressources à grande échelle, comme la pollution de l'air, et sont souvent incapables de résister aux pressions de commerces plus importants, s'effondrant lorsque leurs actifs attirent l'attention d'intérêts économiques de plus grande échelle ${ }^{25}$. Dans une version récente et beaucoup plus vive de cette critique, Brigham Daniels soutient que les études classiques sur les biens communs sont biaisées car elles se font une idée vraiment romantique des anciens modes d'organisation de propriété commune. B. Daniels considère que ces organisations communautaires sont rigides, incapables de suivre le développement technologique moderne, et tendent donc en définitive à réduire le bien-être ${ }^{26}$.

Une autre critique en lien avec celle-ci est plus politique qu'économique. Comme d'autres, j'ai noté que, malgré le fait que de nombreuses normes de propriété communes puissent sembler équitables pour les principaux acteurs de la communauté, ceux-ci ne sont pas les seules personnes impliquées. De nombreuses communautés traditionnelles sont traversées par des niveaux de hiérarchie, et en particulier par des normes relatives aux rôles des hommes et des femmes 27 . Constituant un travail académique majeur en ce sens, Hanoch Dagan et Michael Heller ont appelé de leurs vœux des " communs libéraux " par opposition aux " communs illibéraux " associés à ce que E. Ostrom considérait comme des modes de gestion communautaire efficaces. Ils font valoir que si la prise de décision au sein de la communauté revêt encore une grande importance dans les institutions modernes, telles que les copropriétés, les participants ont besoin d'un débouché, appelé par H. Dagan et M. Heller «sortie». Cette sortie est la capacité d'en partir (même si cette capacité est quelque peu limitée) ${ }^{28}$. Cependant, la question demeure de savoir si une telle option de sortie est compatible avec l'organisation traditionnelle des communs, étant donné que de très nombreux arrangements institutionnels des communs traditionnels mettent l'accent sur la préservation de la continuité et de l'engagement ${ }^{29}$. En tout état de cause, un déficit démocratique continue de hanter les efforts modernes des droits de l'homme visant à protéger les terres et autres biens détenus par les groupes traditionnels ${ }^{30}$. En effet, pour protéger les revendications

25. Carol RosE, «Common Property, Regulatory Property, and Environmental Protection: Comparing Community-Based Management to Tradable Environmental Allowances », in NATIONAL ReSEARCH CounCIL. Committee on the Human Dimensions of Global Change (ed.), Drama of the Commons, op. cit., p. 233-258.

26. Brigham DANIELS, « Emerging Commons and Tragic Institutions », Environmental Law, 37 (3) 2007, p. 515.

27. Carol Rose, «Property's Relation to Human Rights », in Jahel Queralt et Bas VAN DER Vossen (eds.), Economic Liberties and Human Rights, New York: Routledge, 2019, p. 69-91.

28. Michael A. Heller et Hanoch DAGAN, "The Liberal Commons », The Yale Law Journal, 110 (4), 2001, p. 549-623.

29. Henry E. SмIтH, «Semicommon Property Rights and Scattering in the Open Fields », Journal of Legal Studies, 29 (1), 2000, p. 131-170.

30. Sumner B. TwISs, « History, Human Rights, and Globalization », Journal of Religious Ethics, 32 (1), 2004, p. 39-70 ; Madhavi Sunder, "Intellectual Property and Identity Politics: Playing with Fire», Journal of Gender, Race and Justice, 4 (1), 2000, p. 69-98. 
d'une communauté donnée en tant que communauté, il faut laisser libre cours aux méthodes traditionnelles d'allocation de l'accès à la ressource, ce qui signifie également laisser libre cours aux pratiques potentiellement abusives et antidémocratiques au sein de la communauté 31 .

Toutes ces mises en garde concernant les versions communautaires traditionnelles des communs concernent essentiellement un type particulier de communs, à savoir le système de propriété commune limitée, dont les frontières sont délimitées et les règles d'accès contrôlées. En effet, l'accès restreint est l'un des premiers principes de la théorie de E. Ostrom. Mais il existe une autre version très répandue de " communs ", peut-être même assez naïve : les communs comme accès libre. Comme E. Ostrom l'a fait remarquer elle-même, les communs comme accès libre est probablement ce que G. Hardin avait en tête lorsqu'il a qualifié les biens communs de «tragiques ». Mais le libre accès est aussi ce que les spécialistes du droit international traditionnel avaient à l'esprit quand ils ont décrit de très vastes zones de libre accès, en particulier les communs océaniques. Le droit international n'a jamais vraiment cessé de s'intéresser à ce type de très grands communs, comme en témoigne l'intérêt constant au plan international pour les questions de navigation et les revendications des ressources des fonds marins. Au cours des dernières années, les préoccupations relatives aux changements climatiques ont ouvert un nouveau domaine d'intérêt académique pour cette version des communs, à savoir les très grandes ressources communes qui sont ouvertes à tous simplement en raison de leur vaste taille et de leur dimension globale. Les environnementalistes soutiennent maintenant qu'il existe une " confiance atmosphérique " ("atmospheric trust») que les gouvernements sont présumés obligés de préserver dans l'intérêt de tous ${ }^{32}$.

Il existe une autre version des ressources communes en libre accès discutée longuement par les spécialistes actuels. J'ai moi-même joué un rôle dans ce débat, bien que je ne l'ai pas réalisé à l'époque. Il y a quelques années, même avant la publication de Governing the Commons de E. Ostrom, j'avais écrit un article intitulé "The Comedy of the Commons ", dans lequel je tentais de rendre compte de certains types d'espaces qui semblaient avoir défié la qualification de propriété privée pendant de longues années et dans de nombreux endroits. Des institutions juridiques remontant aux Romains ont gardé de tels espaces ouverts au public, bien qu'ils aient été et soient potentiellement clôturables contrairement aux vastes océans ou à l'atmosphère. Autrefois, ces espaces étaient généralement des corridors de transport, en particulier des routes et des voies navigables, mais aussi, dans une certaine mesure, des places et des parcs publics et, plus récemment, des zones de loisirs. Dans la "Comédie », j'ai soutenu que, contrairement à ce qui est le cas pour beaucoup de propriétés, où les frontières exclusives encouragent les investissements et les efforts individuels, nos lois ont gardé ces espaces ouverts en grande partie parce que leur ouverture est plus productive que leur clôture. Le libre accès aux routes, voies navigables et places publiques encourage de plus en plus d'utilisateurs

31. Ibid.

32. Ken Coghill, Charles SAmpford et Tim Smith, Fiduciary Duty and the Atmospheric Trust, Aldershot: Ashgate, 2012. 
et favorise donc ce qu'on appelle maintenant les «effets de réseau » 33 , en particulier le commerce et la communication. Je m'attendais à ce que le même type d'arguments en faveur d'un caractère public ou d'un accès ouvert soit présenté au sujet d'une technologie de communication plus moderne ${ }^{34}$.

La "Comédie des communs" a eu un succès modeste lorsque je l'ai publiée pour la première fois, mais à mon grand étonnement, sa seconde vie a été beaucoup plus longue à l'ère d'Internet. Ce n'était pas de mon fait, mais plutôt grâce aux chercheurs tels que Larry Lessig, Brent Frischman et surtout Yochai Benkler. Ce dernier a fait valoir que l'apprentissage et l'innovation sont généralement mieux encouragés par un "commun de la connaissance" que par la privatisation 35 . D'autres partagent également cette position. L. Lessig, par exemple, utilise des mashups musicaux pour illustrer la façon dont le libre accès stimule la créativité alors que la propriété intellectuelle privatisée peut l'étouffer ${ }^{36}$. Michael Heller et Rebecca Eisenberg affirment qu'une trop grande appropriation des résultats de la recherche scientifique peut conduire à une multiplication de revendications fragmentaires, créant ainsi un "anti commun" qui empêche toute recherche ultérieure ${ }^{37}$. L'argument sous-jacent est qu'un commun en accès libre dans le domaine intellectuel favorise la participation et peut avoir les mêmes effets de réseau que les routes, les voies navigables et les places publiques, augmentant la productivité des artistes et des scientifiques au lieu de la décourager 38 .

Comme je l'ai dit plus haut, Elinor Ostrom elle-même, au cours de sa carrière, s'est beaucoup intéressée à la façon dont l'organisation des systèmes de propriété commune pouvait s'appliquer à un commun de l'information, et son travail a été beaucoup utilisé à l'appui d'arguments contre l'extension d'une appropriation technologique et juridique des avancées intellectuelles. Mais comme l'a souligné Yochai Benkler, le libre accès à la connaissance et à l'information est un système bien plus radical que les communs gérés par les communautés qui sont le lot quotidien des universitaires depuis Governing The Commons de E. Ostrom ${ }^{39}$. Mon impression est que cette différence radicale entre le libre accès et les communs limités n'est généralement pas prise en compte dans la production sur le «copyleft». Les communs limités sont une version reconnaissable d'une propriété circonscrite et exclusive, même si ses frontières englobent une communauté tout entière plutôt

33. Lorsque j'écrivais l'article «La comédie des communs", le terme « effet de réseau » n'était pas réellement employé. Si j'avais su qu'il prendrait cette ampleur, je l'aurais utilisé. Carol Rose, «The Comedy of the Commons: Custom, Commerce, and Inherently Public Property », article cité.

34. ID., "Joseph Sax and the Idea of the Public Trust », Ecology Law Quarterly, 25 (3), 1998, p. 351-362.

35. Yochai BENKLER, "Law, Innovation and Collaboration in Networked Economy and Society", Annual Review of Law and Social Science, 13, 2017, p. 231-250.

36. Lawrence Lessig, Remix: Making Art and Commerce Thrive in the Hybrid Economy, Londres : Bloomsbury Academic, 2008.

37. Michael A. Heller et Rebecca S. EISENBERG, «Can Patents Deter Innovation? The Anticommons in Biomedical Research ", Science, 280 (5364), 1998, p. 698-701.

38. James BoyLe, "The Second Enclosure Movement and the Construction of the Public Domain ", Law and Contemporary Problems, 66, 2003, p. 33-74.

39. Yochai BenkLeR, "Commons and Growth: The Essential Role of Open Commons in Market Economies ", University of Chicago Law Review, 80 (3), 2013, p. 1499-1555. 
qu'un seul individu. Les critères établis par E. Ostrom soulignaient l'importance de la délimitation pour une gouvernance réussie d'une ressource gérée en commun : tel que j'ai décrit ces régimes communautaires de propriété, ce sont des biens communs à l'intérieur, mais des propriétés à l'extérieur 40.

En dépit de certaines caractéristiques qui se chevauchent dans ces deux types de régimes de communs, les communs en tant que libre accès peuvent être profondément perturbateurs des communs fondés sur une communauté organisée. Jamie Boyle, l'un des chefs de file du mouvement " copyleft », s'est insurgé contre l'appropriation des avancées scientifiques faisant rhétoriquement valoir qu'elle serait une nouvelle forme d'enclosure des communs comme on les a connus aux débuts de l'Angleterre moderne. Mais ce que néglige cette analogie, c'est que les premiers communs modernes constituaient eux-mêmes une organisation de la propriété réservée à la communauté qui les utilisait. Par ailleurs, la mise en place de communs en libre accès (une infrastructure de routes et de transports par voie fluviale ou maritime) a en réalité condamné les communs traditionnels. Les nouvelles possibilités de transport et de commerce ont suscité l'intérêt des entrepreneurs qui se sont emparés des communs limités à la communauté pour les transformer en monocultures privées, adaptées à des moutons de pâturage qui fourniraient de la laine pour le commerce international. Ainsi, l'avènement d'un bien commun en libre accès et d'une nouvelle infrastructure de transport étaient les conditions préalables indispensables à la destruction de ces biens communs. Et la terrible vérité, c'est que les communs en libre accès étaient également la condition préalable à la privatisation des anciens biens communs.

Tout cela conduit à s'interroger sur le type de commun qu'ont à l'esprit les spécialistes de propriété intellectuelle hostiles à l'appropriation privée du savoir. Est-ce que ce sont les communs délimités, qui sont des communs du point de vue interne, pour les membres du groupe, mais des propriétés du point de vue externe, pour les personnes qui n'en font pas partie? Ou s'agit-il de communs ouverts à tous les individus et libres d'accès pour tous les nouveaux arrivants? Le professeur Robert Merges ${ }^{41}$ de Berkeley souligne que de nombreux progrès ont été réalisés par des groupes de scientifiques qui partagent des informations et des méthodes tout en résistant à l'empiètement de tiers, en particulier ceux souhaitant monétiser leurs travaux au moyen d'une propriété intellectuelle exclusive. D'autres ont souligné qu'une sorte de commun liée à la collaboration d'un groupe limité est en général au cœur des avancées scientifiques majeures. Après tout, Shakespeare avait besoin de la collaboration de toute la compagnie du Globe Theatre.

L'argument selon lequel l'innovation scientifique et artistique n'est jamais aussi individualiste que nous aimerions peut-être le penser est très largement fondé. Il suggère même que les communs intellectuels idéaux se superposent aux communs

40. Carol M. Rose, «The Several Futures of Property: Of Cyberspace and Folk Tales, Emission Trades and Ecosystems ", Minnesota Law Review, 83 (1), 1998, p. 129-182 mais v. Henry SмITH, "Semicommon Property Rights and Scattering in the Open Fields ", article cité, où il discute de l'attribution interne de biens complexes dans les communs gérés par une communauté.

41. Robert P. MERGES, « Individual Creators in the Cultural Commons », Cornell Law Revue, 95 (4), p. 793-806. 
traditionnellement plus limités qui sont au centre de nombreux ouvrages sur les biens communs. D'autre part, tout comme les institutions traditionnelles ont été critiquées pour leur manque d'ouverture et leur hiérarchisation, les communautés intellectuelles fermées l'ont également été, notamment parce qu'elles peuvent être hostiles aux idées novatrices. Il y a une histoire ici : au XVIII ${ }^{\mathrm{e}}$ siècle, par exemple, le groupe très fermé d'experts en astronomie n'éprouvait que mépris à l'égard des horlogers dont les œuvres réglaient parfaitement le problème de la longitude en mer. Plus récemment, des comités d'octroi de subventions pour la recherche médicale se sont vus reprocher le rejet automatique des projets non conventionnels pour lutter contre des maladies aussi complexes que le sida ou la maladie d'Alzheimer. On pourrait penser que l'une des tâches principales de la politique d'innovation est d'encourager ces deux types de réalisations intellectuelles : les améliorations progressives, d'une part, qui peuvent se produire dans des communs limités de chercheurs et de penseurs ; et, de l'autre, des avancées majeures, qui pourraient nécessiter un accès plus ouvert, ou éventuellement, comme dans le cas du mouvement des enclosures, une propriété privée pour les résultats, peut-être sous la forme de droits d'auteur et de brevets, au moins pour les créations fortement innovantes.

En ce qui concerne les connaissances et les avancées scientifiques, les deux types de communs, en accès limité ou en libre accès, génèrent des problèmes de gouvernance. Les communs limités relatifs à la production d'un groupe sont souvent régis par des normes internes informelles. La question cruciale pour de telles normes concerne l'ouverture à l'expérience et aux informations provenant de sources extérieures. Les communs en libre accès, en revanche, requièrent une forme de gouvernance simplement pour que quelque chose y soit fait. Parfois, les normes peuvent suffire, comme cela a été le cas avec les voyageurs américains du XIX siècle alors qu'ils devaient contourner de profonds trous de boue dans la chaussée. La norme généralement admise était que l'empreinte de pas était autorisée, mais que les intrus ne pouvaient causer que des dommages minimes aux terres adjacentes. En ce qui concerne les connaissances modernes, comme l'a souligné Yochai Benkler ${ }^{42}$, même Wikipedia a besoin de règles. Il en va de même pour la science citoyenne pour laquelle, ceux qui y participent volontairement ont, afin d'être utiles, besoin d'une sorte de collecte de données centralisée pour rendre compte du nombre d'étoiles observées, de leurs mesures de précipitations ou de la date des premières floraisons.

Un exemple particulièrement marquant du besoin de gouvernance dans les plateformes de connaissances en accès ouvert a émergé ces dernières années : la prévention de la participation malveillante sur Internet. Les "fausses informations" (fake news) et le "trolling " viennent perturber la participation politique au sein des systèmes démocratiques. Le rêve de nos plateformes géantes de médias sociaux en libre accès était une sorte de plateforme habermasienne de discours idéal sans contrainte. En fin de compte, c'est aussi le cauchemar, et cette communauté de libre accès attend toujours un catéchisme pour « gouverner les communs ».

42. Yochai BENKLER, « Degrees of Freedom, Dimensions of Power », Daedalus, 145 (1), 2016, p. 18-32. 


\section{Conclusion. Quelques sujets pour la poursuite de la recherche}

L'étude des communs au cours des dernières décennies a connu quelques rebondissements inattendus que je n'ai pas discutés ou n'ai pas mentionnés. Par exemple, les travaux d'Henry Smith sur les "semi-communs " suggèrent que les communs traditionnels - les domaines communs de l'Angleterre - étaient non seulement strictement régis par des normes internes, mais étaient également l'objet de revendications de propriété privée dont les participants étaient parfaitement conscients. Elinor Ostrom a nié que les biens communs gérés nécessitaient un Léviathan ou la propriété privée, comme l'a postulé G. Hardin, mais le travail de H. Smith souligne que les communs traditionnels étaient en réalité une combinaison de Léviathan et de propriété privée, bien qu'à petite échelle.

G. Hardin et E. Ostrom ont ainsi tous deux reconnu que les ressources communes pouvaient mener à la tragédie, mais qu'elles pouvaient aussi être gouvernées d'une manière ou d'une autre. Mais comment cela se produit-il ? Il y a quelques années, James Krier a souligné avec vigueur que la gouvernance des communs est ce qu'il appelle "La tragédie des communs, deuxième partie » 43. En d'autres termes, la création d'un système de gouvernance est en lui-même un problème de communs ou d'action collective secondaire, avec toutes les incitations à se soustraire de la participation et à profiter des avantages sans y contribuer.

Une question importante pour des recherches à approfondir est de savoir s'il serait plus facile de résoudre le problème de l'action collective secondaire - dans la création et le maintien de la gouvernance des ressources communes - que de résoudre le problème de l'action collective primaire dans l'utilisation des ressources. Après tout, la création de règles pour l'utilisation des biens communs pourrait être une question moins brûlante que de tenter d'arrêter directement la surutilisation en l'absence de règles. Les travaux d'Henry Smith sur les semi-communs suggèrent que ce pourrait bien être le cas. Il note qu'au début du système anglais de domaine commun, les agriculteurs du Moyen Âge sont effectivement parvenus à se mettre d'accord entre eux pour gérer leurs champs en commun. Autrement dit, ils ont vu l'avantage qu'il y avait à créer un commun et ils ont créé une structure organisationnelle avant d'utiliser réellement la ressource. D'autre part, Gary Libecap, travaillant sur un problème beaucoup plus moderne - les grandes réserves souterraines de pétrole et de gaz -, a fait valoir que "l'uniformisation » (" unitization») des règles régissant les propriétaires terriens du sol serait un moyen très efficace d'exploiter cette ressource. Mais ses recherches montrent qu'en pratique les propriétaires fonciers sont contraints de créer ces règles à travers une sorte " de voile d'ignorance" lorsque les participants ne connaissent pas la quantité de pétrole et de gaz qui est stockée sous leurs propriétés individuelles. Une fois qu'ils savent que le pétrole leur est accessible, ils ne veulent pas le partager. Mais s'ils en sont au

43. James E. KrIER, « Tragedy of the Commons, Part Two », Harvard Journal of Law, 15 (2), 1996, p. 325-347. E. Ostrom avait connaissance de ce deuxième versant des problèmes liés aux communs. Bien qu'elle ne l'ait pas traité en tant que tel, elle y apporte des solutions dans ses principes généraux. 
stade où ils ne connaissent pas encore la quantité en réserve, personne n'en sait suffisamment sur la ressource pour prendre la peine de tenter de la gérer ${ }^{44}$.

Le problème des réserves de pétrole est très étroitement lié à un autre problème de communs ou d'action collective : à quel moment savons-nous qu'il s'agit d'une question de ressource commune ? Cette question est particulièrement problématique dans les domaines de l'environnement. L'épuisement ou la pollution de la ressource peut se produire avant même que nous le remarquions. Et si une ressource est vraiment un commun, ouvert à beaucoup d'autres, aucun individu ne souhaite investir dans l'apprentissage de sa dégradation ou s'intéresser aux méthodes de récupération potentielle. L'apprentissage n'est qu'une autre forme d'investissement, et contrairement au cas des biens privés, l'investissement dans la recherche relative aux ressources communes est faible, parce que d'autres sont susceptibles de partager les résultats d'une connaissance plus ample de la ressource commune. C'est pourquoi l'apprentissage des problèmes communs, en particulier des communs de grande envergure, reproduit le problème des communs. C'est une des raisons pour lesquelles les problèmes environnementaux nous rattrapent souvent par surprise. Nous ne les discernons qu'après qu'ils sont devenus très sérieux parce que jusque-là nous n'étions pas suffisamment motivés pour leur prêter attention. Les futures recherches relatives à cette question de l'apprentissage pourraient toutefois apporter quelques améliorations 45 . Certains problèmes de ressources communes peuvent être plus facilement observés que d'autres : l'épuisement des forêts, par exemple, en comparaison avec la surcharge de l'air par le dioxyde de carbone. La recherche sur cette question - quel genre de problème commun est en cause ? - pourra nous aider à nous concentrer sur l'investissement dans la connaissance des problèmes plus aigus et les plus difficiles concernant les biens communs.

Un autre domaine de recherche potentiel découle de l'observation faite il y a plusieurs décennies par Russell Hardin (sans rapport avec Garrett) : à savoir que la tragédie des communs est vraiment un dilemme du prisonnier comme certains l'ont appelée. C'est maintenant une opinion largement acceptée. Pourtant, on pourrait bien se demander si et comment le plus grand dilemme (ou tragédie) peut différer du dilemme conventionnel à deux personnes. Les problèmes de méfiance entre deux partenaires commerciaux potentiels sont-ils vraiment semblables à ceux des pays qui tentent de s'entendre sur la réduction des gaz à effet de serre ? Dans ce dernier cas, la méfiance estelle l'enjeu principal, plutôt que les désaccords sur la répartition des coûts?

Toutes ces questions nous donnent du grain à moudre pour de nouvelles recherches. Garrett Hardin nous a laissé la formule accrocheuse de la «tragédie des biens communs" et adressé une mise en garde concernant la dégradation des ressources communes. E. Ostrom nous a convaincus que ces ressources peuvent être étudiées et gérées de façon fructueuse. La recherche sur les communs s'étend bien au-delà de ces deux auteurs, mais il n'est peut-être pas surprenant que les connaissances actuelles résonnent en écho à la fois proche et lointain à ces deux pionniers, même lorsqu'elles relèvent de sujets pratiquement inconnus au moment de leurs travaux.

44. Gary D. LiBECAP, Contracting for Property Rights, Cambridge : Cambridge University Press, 1989.

45. Carol Rose, « Surprising Commons », Brigham Young University Law Review, 6, 2014, p. 1257-1282. 


\section{- L'autrice}

Carol M. Rose est professeure émérite à l'Université de Yale. Elle a récemment publié : - Property and Persuasion: Essays on the History, Theory, and Rhetoric of Ownership, Abingdon, Oxon : Routledge, 2019 [publication originale en 1994].

Pour plus de précisions, voir supra la «Présentation » de l'article. 
Des justiciables inégaux ?

coordonné par Aude Lejeune et Alexis Spire

Aude Lejeune, Alexis Spire

Inégalités sociales et judiciaires face au tribunal. Présentation du dossier

Camille François

Déférer au tribunal. Les figures imposées de la défense des locataires au tribunal des expulsions

Émilie Biland, Sibylle Gollac,

Hélène Oehmichen, Nicolas Rafin,

Hélène Steinmetz

Frédéric Salin

La classe, le genre, le territoire :

les inégalités procédurales dans la justice familiale

Hugo Wajnsztok

Inégalités sociales et judiciaires aux prud'hommes : le cas des référés

Des indemnisations inégales. Recours à un.e avocat.e et variations des sommes accordées aux victimes

Bartolomeo Cappellina, Cécile Vigour

Recours différenciés au droit et aux tribunaux civils. Étude des représentations et modes de réception de la justice

Le devoir de vigilance des entreprises transnationales

coordonné par Luca d'Ambrosio et Pauline Barraud de Lagerie

Luca d'Ambrosio,

Pauline Barraud de Lagerie

Luca d'Ambrosio

Guillaume Delalieux

Armand Hatchuel, Blanche Segrestin

Tatiana Sachs, Juliette Tricot

Pauline Barraud de Lagerie,

Élodie Béthoux, Arnaud Mias,

Élise Penalva-Icher
La responsabilité des entreprises reformulée par la loi : un regard pluridisciplinaire. Présentation du dossier

Le devoir de vigilance : une innovation juridique entre continuités et ruptures

La loi sur le devoir de vigilance des sociétés multinationales : parcours d'une loi improbable

Devoir de vigilance : la norme de gestion comme source de droit? La loi sur le devoir de vigilance : un modèle pour (re)penser la responsabilité des entreprises

La mise en cuvre du devoir de vigilance : une managérialisation de la loi?

\section{Questions en débat'}

\section{Anne Deysine}

Jean-Guy Belley
La cour Roberts et le retour du gouvernement des juges?

Ubi communitas, ibi ius?

\section{Traduit pour vous}

